\title{
Novedades emergentes: una propuesta naturalista al aprendizaje práctico*
} Emergent Novelties: A Naturalistic Proposal to Motor Learning

\author{
Ximena A. González Grandón ${ }^{\dagger \neq}$
}

\begin{abstract}
Resumen
En este artículo propongo un marco epistémico para integrar el pensamiento emergentista y auto-organizado a los estudios del aprendizaje de nuevo conocimiento práctico. Particularmente, se considera que desde una epistemología relacional y dinámica, los patrones de actividad emergentes y auto-organizados son condiciones necesarias para que surja la novedad práctica y se vuelva parte del repertorio de habilidades prácticas. De acuerdo a la perspectiva de sostén, el punto de partida es la interacción dinámica del agente corporizado con su entorno donde los patrones de actividad se consideran propiedades relacionales del sistema. Se pone particular atención a la normatividad de la novedad práctica descrita en dos aspectos -biológico y social-, y demarcada de una normatividad proposicional. Asimismo, se introduce la noción de novedad práctica como una definición operacional desde el modelamiento con la teoría de sistemas dinámicos. Por último, se asume que el desarrollo ontogenético es una variable crucial para la adquisición de la novedad práctica.
\end{abstract}

Palabras clave: conocimiento práctico - novedad - emergencia - auto-organización - aprendizaje motor

\begin{abstract}
In this article an epistemological viewpoint is proposed in order to integrate emergent and self-organized thought with studies on learning new motor knowledge. Particularly, it is considered that from a relational and dynamical epistemology the emergent and self-organized activity patterns are necessary conditions for practical novelty arises and become part of human agent's repertoire of motor skills. According to the approach sustained here, the starting point is the dynamical interaction between the embodied agent and the environment, where those activity patterns are considered relational properties. Particular attention will be paid to the description of the normativity of two aspectsbiological and social-, and from distinguish it from a propositional normativity. Furthermore, an operational notion of practical novelty will be introduced, that comes from the modeling with DST. Finally, it is assumed that ontogenetic development plays a crucial role as a valuable variable for the acquisition of practical novelty.
\end{abstract}

Keywords: know how - novelty - emergence - self-organization - motor learning

\footnotetext{
* Recibido: 2 de Mayo de 2014. Aceptado en versión revisada: 13 de Agosto de 2014.

† Investigación Posdoctoral en el Instituto de Filosofía y Ciencias de la Complejidad (IFICC), Chile. Académica Facultad de MedicinaUNAM, México. Para contactar a la autora, por favor, escribir a: glezgrandon@gmail.com.

* Este artículo es resultado del trabajo del Posdoctorado Fondecyt No 3150704 "Los sentimientos epistémicos, su naturaleza y normatividad desde una aproximación corporizada y situada”, Chile. Participa de los proyectos CB 182084 de CONACYT y PAPIIT IN401315, México.

Metatheoria 5(1)(2014): 129-143. ISSN 1853-2322.

(C) Editorial de la Universidad Nacional de Tres de Febrero. Publicado en la República Argentina.
} 


\section{Introducción}

Al menos dos diferentes aproximaciones al aprendizaje humano proponen explicaciones coherentes. Por un parte, la caracterización filosófica de Jerry Fodor $(1980,1981)$ que se remonta a la tradición platónica e introduce el concepto de conocimiento proposicional nuevo por re-ordenamiento de los conocimientos anteriores. Por otra parte, la explicación por estadios de la psicología del desarrollo proveniente de los trabajos de Piaget (1959) y Gesell (1946), que subraya la correlación entre edades específicas y el aprendizaje por resolución de tareas cognitivas. Pese a que estas dos formas de aproximación resultan muy útiles para resolver algunos problemas, dejan fuera otros. Por ejemplo, la existencia de novedad en ámbitos no proposicionales, la influencia de los procesos ontogenéticos en la adquisición de nuevos conocimientos o respecto de los posibles mecanismos involucrados en la generación de novedad.

En este artículo, queremos proponer una epistemología que integre el pensamiento emergentista y auto-organizado con estudios naturalistas del aprendizaje de un nuevo conocimiento práctico. Se considera que los agentes humanos son organismos corporizados que están organizados para mantenerse fuera del equilibrio termodinámico y que cambian constantemente al interactuar con el medio y sus perturbaciones. En cada cambio emergen nuevos procesos en el agente que no pueden reducirse a los procesos de los cuales provienen. De alguna manera, estos procesos se están reorganizando cotidianamente dado que los agentes actúan, se desarrollan ontogenéticamente y aprenden todos los días en contextos particulares. Una de las formas de cambio es la adquisición de conocimiento práctico legítimo, como al reaccionar espontáneamente de una caída o al llevar a cabo una práctica intencionada y disciplinada, que parece una forma de aprendizaje novedoso que no involucra una normatividad de contenidos semánticos. La pregunta que surge es cómo caracterizar ese ensamble de patrones de actividad práctica que se adquiere como conocimiento normado y cambia con el tiempo. En este trabajo, se considera que la teoría de sistemas dinámicos (TSD) es una herramienta útil cuando se quiere explicar el proceso a través del cual un agente humano adquiere conocimiento práctico y puede dar respuesta a la pregunta anterior al resolver problemas que otros marcos explicativos no resuelven. Más concretamente, se propone que los patrones de actividad en la adquisición de un nuevo conocimiento motor, tiene lugar a través de procesos emergentes y autoorganizados que pueden ser explicados si (a) se atiende el rol normativo y ontogenético del agente, (b) se parte de un marco epistémico relacional y dinámico, y (c) se toman en cuenta evidencias experimentales de aprendizajes motores utilizando la TSD (Thelen \& Corbetta 2002, 2013, Kelso 2014).

En la primera parte, se dan dos ejemplos clásicos del estudio de la novedad, el filosófico y el psicológico. Estos dos ejemplos, nos sirven para hacer notar los huecos explicativos que pueden ser subsanados con propuestas dinámicas y auto-organizadas. En la segunda parte, se plantea que la normatividad del conocimiento práctico es distinta a la del conocimiento proposicional. Lo que da lugar para proponer una epistemología relacional y activa cuyo énfasis es la interacción práctica entre el agente corporizado y situado en un contexto. Desde este sostén, se arguye que el aprendizaje del nuevo conocimiento práctico de una habilidad motora presenta una normatividad que puede describirse a partir de dos aspectos: por las posibilidades y constricciones corporales para llevar a cabo una acción, y por la normatividad social que plantea los consensos intersubjetivos para realizar prácticas adecuadas. En la tercera parte, se propone una caracterización de la novedad práctica desde el marco propuesto, y se describe un posible mecanismo a partir del cual emerge la novedad utilizando las herramientas epistémicas y conceptuales de la teoría de sistemas dinámicos. En concreto, se introduce una explicación emergentista donde la auto-organización de los sistemas es una condición necesaria para la generación de novedad práctica. Por último se muestra la necesidad de la ontogenia humana como una variable crucial para entender la novedad práctica. Así, el sentido en el que podemos decir que la novedad práctica se aprende de manera emergente, no puede formularse con independencia de un marco epistémico dinámico y auto-organizado. 


\section{Antecedentes teóricos y empíricos}

Durante las décadas de los 80's y 90's se produjo un interesante debate entre los teóricos del aprendizaje en cuanto a la mejor forma de conceptualizar la generación de novedad entre los individuos humanos. La "paradoja del aprendizaje", como llaman algunos al conjunto de argumentos al respecto que proviene desde tiempos platónicos, ${ }^{1}$ plantea que el conocimiento nuevo no puede derivar completamente del conocimiento viejo, dado que no sería nuevo; pero, la nueva parte del conocimiento tampoco puede ser completamente nueva, porque si no nunca podría entenderse. En cuanto a esto, en la filosofía cognitivista es famosa la tesis de Jerry Fodor (1980) de la imposibilidad de aprender algo genuinamente nuevo, dado que no pueden surgir nuevas estructuras cognitivas. La razón estriba en que, de modo esencial, todas las estructuras necesarias para el conocimiento están presentes desde el nacimiento. Esta manera de plantear las cosas supone precondiciones y mecanismos innatos que den cuenta de la génesis de la novedad en el terreno proposicional. Bajo este supuesto, la novedad de un conocimiento es un re-ordenamiento de representaciones existentes comandada por el procesador central que resulta en una actualización de representaciones previas. En este caso, la causa eficiente de una nueva creencia adquirida proviene de la relación lógica con una creencia primitiva o innata (Fodor 1981, Viale 2013, Witt 2005). Un problema que ha sido formulado de distintas maneras por los críticos de esta propuesta es que los eventos mentales son reducidos a casos de determinación causal o funcional, y están únicamente constituidos por representaciones internas (objetos proposicionales con valor de verdad) (Boom 2012). Además, la paradoja sigue: no hay posibilidad de nuevo conocimiento primitivo, la novedad tiene la misma cualidad de la representación simbólica de la cual proviene, solo que se actualiza en sujeción a las mismas leyes (Anaxágoras [en Burnet 1920], Fodor 1980, 1981, Platón 1871). Siguiendo esta tradición filosófica, Ulrich Witt (2005) plantea:

La novedad mental implica: 1) información potencialmente contenida en la más simple red proposicional que permite la recombinación de elementos heterogéneos. 2) Nuevas ideas o proposiciones que resultan de un proceso en el cual los elementos previamente conocidos (sujetos, predicados u objetos) son combinados dentro de una extensión combinatoria. 3) Dada la caracterización genérica como una extensión combinatoria, el proceso de generación puede ser trasladado a un algoritmo numérico que corre en un programa de computadora (Witt 2005, p. 128).

Este sostén teórico proposicionalista, también es tomado en cuenta por la teoría computacional de la mente. Wolpert, Ghahramani y Flanagan (2001), desde esta perspectiva, proponen un modelo de aprendizaje: "Internal forward dynamic model". En este modelo, el aprendizaje de novedad se explica desde una función comandada por el procesador central que ordena cuales novedades deben mantenerse para poder predecir las consecuencias sensoriales de una acción particular. Además, el procesador central conoce lo que es apropiado o no apropiado para diferentes tareas y ambientes. De hecho, es necesario para estimar las órdenes motoras requeridas, para archivar las respuestas sensoriales deseadas y para actualizarse constantemente gracias a los nuevos aprendizajes.

Ambas perspectivas (cognitivismo y TCM) han tenido gran utilidad en construir explicaciones internalistas que niegan que la mente humana surja como un tabula rasa, han dirigido hipótesis experimentales muy coherentes, como es el caso de explicaciones lingüísticas que proponen la idea de conjuntos casi infinitos de combinaciones de palabras. Además, son estas perspectivas las que han promovido una base empírica fisiológica donde la generación de novedad proviene del robustecimiento de conexiones neurales. Sin embargo, en cualquiera de estas explicaciones, no queda claro si existe como tal una forma de novedad que vaya más allá del terreno lingüístico o proposicional, por ejemplo, no ha sido muy explorado si existe una novedad en el conocimiento práctico. Tampoco están interesados en entender cómo es que suceden esos re-ordenamientos o sí de hecho podría haber un elemento completamente nuevo posterior al aprendizaje. Como veremos en los siguientes

\footnotetext{
${ }^{1}$ En el diálogo platónico "Menón o de la virutd", se plantea la paradoja del aprendizaje para argumentar que el aprendizaje es una actividad de los individuos por sí mismos, que depende del conocimiento previo, de lo que ya se conoce, por tanto el aprendizaje de algo nuevo es imposible. En la tradición platónica el alma inmortal ya conoce todo antes de que el individuo nazca, por lo que el aprendizaje de la novedad es sólo una cuestión de recolección y activación de conocimientos previos (Platón 1871).
} 
apartados, existen otras perspectivas que dan cuenta de estos elementos.

Otro ejemplo clásico al estudio de la novedad en el aprendizaje, es la perspectiva de la psicología del desarrollo. Entre sus líneas de investigación se buscan las edades en las cuales los infantes prelingüísticos y lingüísticos resuelven tareas cognitivas específicas. Instancias paradigmáticas son las etapas clásicas del desarrollo cognitivo de Jean Piaget (1959) y los estadios del desarrollo de Arnold Gesell (1946). A diferencia de la aproximación filosófica cognitivista, en la psicología del desarrollo siempre ha existido un interés por el conocimiento práctico, de una manera explícita o implícita, y por los patrones de comportamiento. De hecho, es el caso de las teorías del aprendizaje motor donde predomina el intento por determinar una sola función del cambio conductual a través de distintas tareas. Por ejemplo en el modelo psicológico-matemático propuesto por James Mazur y Reid Hastie (1978), el aprendizaje se mide por una sola función de cambio al resolver una sola tarea siguiendo la "ley de aprendizaje de los dominios cognitivos y motores" (un proceso de acumulación de información con una tendencia constante a responder correctamente con la práctica).

Algunas críticas comunes a este tipo de enfoque psicológico son las siguientes:

1. No están interesados en conocer cómo es que ocurren los procesos de cambio o de novedad en distintos niveles y a diferentes escalas de tiempo, sino solamente en medir cuando se resuelven metas cognitivas, sensoriomotoras o sociales.

2. Consideran que el objetivo es la resolución correcta de la tarea y por lo tanto los errores durante el proceso de aprendizaje son considerados elementos negativos y no suelen ser medidos.

3. Sus datos empíricos suelen obtenerse de mediciones que se avocan a tareas que se encuentran fuera de contexto y se enfocan solo en el grupo motor específico a la conducta medida, no en todos los sistemas motores involucrados, ni es las muchas las funciones de cambio.

Ahora bien, la crítica depende de asumir que la novedad práctica solo deviene de un mecanismo solitario al resolver correctamente una tarea única donde el resto de sistemas del organismo no juegan un papel constitutivo. Como veremos más adelante, si modelamos la novedad práctica como un proceso dinámico es fácil ver el rol que juega el resto de sistemas del organismo.

Bajo estos dos ejemplos de perspectivas, filosófica-computacional y psicológica, se sugiere que el aprendizaje correcto se busca en el terreno proposicional o en la solución de una tarea específica. No obstante, el interés de este artículo no es entender cuáles representaciones simbólicas subyacen al aprendizaje, ni mucho menos cuál es el rango de edad para resolver una tarea práctica. Más bien, cómo se puede explicar el carácter práctico e interactivo de una novedad y cuál es la relación de ésta con formas de normatividad distintas a la proposicional.

\section{Conocimiento práctico y su normatividad}

El aprendizaje es una cuestión normativa. Para que un agente humano pueda interactuar de un modo correcto con el mundo, es necesario, dado que el mundo no es un lugar completamente predecible, que el agente aprenda las mejores maneras de interactuar con él. Además de habilidades conceptuales, la transmisión y adquisición del conocimiento práctico es una necesidad para la convivencia con el ambiente, para reaccionar adecuadamente a un acto inesperado o para perfeccionar una habilidad motora siguiendo los patrones normados del acuerdo intersubjetivo.

Sobre el problema de cómo caracterizar el conocimiento práctico y su normatividad se ha escrito mucho. Por lo general esto se ha hecho bajo el supuesto de un contraste con el conocimiento proposicional que presupone una jerarquía menor a la habilidad motora y un análisis dependiente del proposicional. No obstante, hay quienes defienden la importancia del conocimiento práctico como una dimensión no inferior sino autónoma. Este tipo de conocimiento estriba en saber cómo hacer algo, por ejemplo, saber caminar o saber escribir. De un modo más formal, consiste en tener la 
habilidad de hacer algo bajo determinadas circunstancias (por ejemplo, para saber caminar es necesario tener piernas y poder moverlas). Gilbert Ryle (1946) distinguió este tipo de conocimiento con clara independencia respecto al conocimiento proposicional: saber cómo hacer algo no es sólo tener creencias correctas acerca de cómo hacerlo, más bien, es la acción adecuada o no adecuada.

Para los fines operacionales de este artículo, saber hacer algo no es meramente poder hacerlo, sino ser capaz de hacer algo de forma exitosa o correcta al intentarlo de acuerdo a ciertas reglas: teniendo viabilidad de las posibilidades de movimiento y siguiendo las reglas de comportamiento de una disciplina cultural particular. Podemos estar de acuerdo con la conceptualización de Ryle, pero lo que me interesa hacer notar en este texto, es que se requiere tener un modelo plausible que tome en cuenta una normatividad que no tiene que ver con corrección de enunciados, sino con dejar de explicar la normatividad práctica desde un proposicionalismo. El problema de demarcar lo práctico de lo semántico, es indudablemente un problema importante que no va a ser fácilmente resuelto por teorías cognitivistas-computacionalistas. Más bien, requiere tomarse en serio que la epistemología es relacional, es decir, que el agente humano interactúa con un entorno a través de prácticas corporizadas no internalistas, que adquieren su significado en la práctica no de manera pre-dada. En otras palabras, mi sugerencia es que este problema requiere incorporar, desde el inicio, recursos explicativos de las interacciones y coordinaciones que cambian entre el agente humano y su entorno.

Desde esta epistemología relacional (muy vinculada a las aproximaciones corporizadas y ecológicas a la cognición humana), el acoplamiento histórico entre las características biológicas del agente y el entorno ecológico, da lugar para pensar en normas de al menos dos aspectos distintos no vinculados directamente a la cuestión proposicional. Primero, el aspecto corporal, las constricciones biológicas como los arcos de movilidad y su resistencia, se muestran como características que se comparten como especie para la realización de cualquier práctica motora. El agente humano no puede volar, pero puede caminar y correr. No hay duda que existen mejores maneras que otras, las cuales están relacionadas con las mejores posibilidades de movimiento que tiene el agente dependiendo las condiciones que impone el entorno y qué él se impone a sí mismo. Como los patrones de movimiento que están organizados en grupos musculoesqueléticos que se coordinan de modo sinérgico para poder respirar, mamar, parpadear o agarrar de la mejor manera. Y además, la práctica constante de estas habilidades puede provocar cambios estructurales en el agente, como aumentar el tamaño de los músculos utilizados (hipertrofia) o la forma de la postura. Esto no implica que siempre se aprenda la práctica más adecuada, a veces se puede aprender mal o la enseñanza puede ser incorrecta y el agente corporizado adquiere una habilidad motora inadecuada. Igualmente, las adecuadas o inadecuadas posibilidades de interactuar con el entorno son muy diversas, lo que puede depender de la etapa de desarrollo en la cual se encuentre un agente. No va a caminar de igual manera un infante de un año, un adulto de 34 o un anciano de 92. De hecho, los bebes menores de 10 meses no tienen la capacidad de caminar, por la postura, por la inmadurez de los arcos de flexión de la cadera, y por la fuerza en las piernas para vencer la fuerza de gravedad. Este tipo de caracterizaciones como parte del aspecto biológico o corporal de la normatividad, da cabida a la consideración de la diversidad de formas de adquirir la novedad práctica. El proceso va a ser distinto dependiendo la edad de desarrollo del agente y las condiciones de posibilidad de su actividad motora. Más adelante se analiza con mayor profundidad esta idea. Para terminar con este apartado, solo resta mencionar que las conceptualizaciones de affordances de James Gibson (1977), de intencionalidad corporal de Merleau-Ponty (1975) y de hacer sentido de Varela, Thompson y Rosch (1991) toman en cuenta este tipo de normatividad corporal, y han sido retomadas desde muchos campos.

Recapitulando, si un agente adquiere un nuevo aprendizaje motor, tomando en cuenta este primer aspecto de la normatividad, no tiene que ver con aprender conceptos verdaderos, sino con aprender la habilidad de ajustar los constituyentes de la acción que están involucrados en la actividad: los ritmos de respiración o la postura del cuerpo y de los miembros involucrados, para poder adaptarse a las perturbaciones del entorno y a su condición endógena.

El segundo aspecto tiene que ver con la normatividad social que considera una práctica exitosa, esto recalca el papel que juega el contexto situado de grupos sociales y ambientes específicos en el que 
se encuentra el agente. Dicho de otra manera, una práctica motora es adecuada o correcta o precisa, dependiendo los estándares sociales. Esto lleva a pensar, que las adscripciones normativas del saber cómo se garantizan únicamente cuando la ejecución de un agente encuentra ciertos estándares de eficiencia o de éxito según el contexto cultural particular. Así, saber cómo llevar a cabo una actividad requiere el desarrollo de una cierta técnica a través del tiempo, donde se implican episodios diacrónicos en los cuales el entrenamiento juega un rol central. ${ }^{2}$ Pero cómo en el caso de la normatividad corporal, la justificación normativa de una práctica motora no sucede por la apreciación de las buenas razones en un marco semántico. Los estándares motores se derivan de patrones de actividad que promueven un comportamiento habilidoso de cierto nivel de competencia. Las reglas no son proposiciones correctas sino planes motores adecuados que se aprenden intencionada o desintencionadamente. Como plantea Josefa Toribio $(2008),{ }^{3}$ la práctica no se justifica normativamente a partir del conocimiento de las proposiciones conceptuales, sino de un saber cómo consensuado en un entorno singular de normas motoras particulares. La cultura humana está llena de cánones de diversas disciplinas prácticas alrededor del mundo, aprenderlas suele ser una tarea de perfeccionamiento y adaptación durante largos periodos de la vida que puede resultar en la generación de experticia.

Así, los dos aspectos de la normatividad práctica se vinculan: un agente sabe cómo llevar a cabo una acción motora cuando tiene las posibilidades motoras para realizarlo y está acorde a las normas de una sociedad particular.

Sobre este punto, Dreyfus (2002) también hace un planteamiento:

La adquisición de habilidades confirma que cuando uno adquiere experticia, el saber cómo adquirido se experimenta como discriminaciones cada vez más finas de situaciones que son equiparadas con la respuesta apropiada a cada una. La “aprehensión máxima” ('Maximal grip') de Merleau Ponty nombra la tendencia corporal a refinar su respuestas para traer la situación actual más cercana a una gestalt óptima. Así, el aprendizaje exitoso y la acción no requieren representaciones proposicionales mentales. $\mathrm{Ni}$ tampoco requieren representaciones cerebrales semánticamente interpretables (Dreyfus 2002, p. 367).

Desde un enfoque relacional, dejamos atrás una sobre-intelectualización del conocimiento práctico y se sugiere que la normatividad de dos aspectos es acerca de la convergencia entre la tendencia corporal asociada a las posibilidades de movimiento que tiene el cuerpo humano dependiendo la etapa ontogenética en la que se encuentre. Así como del contexto de normas sociales en el que esté situado el agente. Una vez que aceptamos esta caracterización de conocimiento práctico y su doble normatividad como un recurso explicativo útil, podemos llegar al problema central que nos interesa.

\section{Novedad del conocimiento práctico}

¿Cómo caracterizar la novedad práctica desde el punto de vista relacional con posibilidad de ser científicamente modelable? En este apartado voy a enfocarme en intentar responder a este cuestionamiento a través de un modelo explicativo de los sistemas dinámicos. Voy a partir con una definición operacional del aprendizaje de la novedad práctica:

La novedad P es un patrón emergente generado por la producción interna de un nuevo elemento del sistema.

Donde un patrón emergente se considera una organización que surge por el comportamiento colectivo y organizado de los componentes de los sistemas involucrados, que no ha sido previamente experimentado por el agente cognitivo, y que se puede describir como un elemento nuevo en el tiempo que no puede reducirse al estadio del que proviene. Así, descriptivamente hablando, el patrón

\footnotetext{
${ }^{2}$ Lo que no niega que puedan existir casos de personas que son virtuosas o muy capaces de realizar una actividad particular sin necesidad de práctica previa.

${ }^{3}$ Los conceptualistas como McDowell (1994) y Brewer (2006), argumentan que las razones para que el sujeto actúe no pueden venir de contenidos no conceptuales, ya que el sujeto no tendría razones por las cuales actuar y ni criterios suficientes para la justificación del conocimiento. Al respecto, Susan Hurley (2008) opina que las razones conceptuales para la experiencia del sujeto solo ocurren si se tiene una visión sobreintelectualizada de la mente y se asume que la racionalidad práctica es solo una forma de racionalidad conceptual.
} 
emergente es parte de un nivel impredecible y no deducible desde el nivel que lo antecede, y surge como inestable en el tiempo y respecto de perturbaciones externas. De hecho, hay bastante consenso respecto a la idea que esta nueva inestabilidad de los sistemas internos puede correlacionarse con el tipo de actividad motora que surge en un agente. La emergencia sucede en el plano temporal, primero emerge la inestabilidad y después el sistema se re-organiza y se vuelve a generar una estabilidad. Se plantea que el agente efectivamente ha aprendido una nueva habilidad motora cuando el sistema vuelve a adquirir estabilidad.

Ahora bien, la definición propuesta se vincula con el punto de vista sistémico, dado que una habilidad motora es el producto de muchos sistemas trabajando de manera conjunta o disjunta. Por ello, se hace difícil plantear que sea solo un proceso o una sola función de un órgano determinado. Por ejemplo, si pensamos en la habilidad motora de caminar, estamos hablando de procesos complejos que involucran la coordinación de muchos componentes en conjunto: coordinación de sistemas visuomotores de las piernas, el tronco y los brazos, coordinación musculoesquelética, de ligamentos y tendones, así como el control de la posición y el equilibrio con otras partes del cuerpo, la postura particular- el monitoreo metacognitivo, las transformaciones cinemáticas para compaginar las distintas distancias, las transformaciones dinámicas como las fuerzas que se aplican a distintos movimientos a diversas distancias (Hofsten \& Fazel-Zandy 1984, Rochat 1992). Al plantear esta base empírica (corporal-fisiológica) para una tarea tan compleja como la habilidad motora de caminar, se torna más intuitivo partir de un marco sistémico y auto-organizado para entender el conocimiento práctico. El marco sistémico considera la novedad práctica resultado de múltiples sistemas que interactúan: operaciones a un nivel metacognitivo, afectivo, motoro o de memoria, y que generan en su interacción un nuevo patrón de comportamiento o no lo generan. Si lo generan, estamos hablando de que la novedad práctica ha sido aprendida y persiste en el tiempo de manera estable.

Y el marco auto-organizado permite pensar que la novedad se organiza a sí misma por la interacción entre ciertos sistemas. Los ensambles de patrones auto-organizados son resultado del proceso continuo de modificaciones y alteraciones de las habilidades presentes del agente que evolucionan en el tiempo. La novedad sucede en agentes que cambian, ellos mismos y su entorno de interacción. Pero la auto-organización no ocurre a un solo nivel, sino a varios, lo que posibilita la descripción en escalas de tiempo. De hecho, la explicación de aprendizaje práctico puede darse en distintos niveles temporales: (1) cuando el agente no tiene el conocimiento, (2) cuando emerge la inestabilidad, o (3) cuando se auto-organiza la nueva estabilidad y se adquiere o no del conocimiento.

A partir de estas características, se posibilita proponer un modelo dinámico respecto a la emergencia de la novedad y su adquisición en el tiempo, donde:

1. Los patrones temporales sistémicos se vuelvan inestables. Esto es, que la coherencia del patrón comúnmente utilizado y actual sea interrumpido.

2. El sistema vuelva a re-organizarse a una nueva configuración y si esto acontece, el sistema aprendió a partir de la emergencia de un nuevo elemento que no puede reducirse a los patrones sistémicos anteriores.

Alrededor de 20 años atrás, Siegler (1996) planteó una idea similar al sugerir que el aprendizaje busca generar inestabilidad para que de ese nuevo estado, emerja una nueva auto-organización. Siguiendo este punto de vista y con una intención similar en cuanto a crear modelos naturalistas de la novedad práctica en el aprendizaje, algunos teóricos han apuntado hacia la idea de la transición como condición necesaria para que se aprenda una habilidad motora particular (Corbetta \& Thelen 2013, Kelso 2014). A pesar de que todavía no se tienen todas las herramientas metodológicas y experimentales para poder medir todos los procesos temporales involucrados que son parte de la actividad motora -como las operaciones en niveles meta-cognitivos que seleccionan las mejores estrategias, recuerdos previos en situaciones similares, automatismos o movimientos involuntarios-. Utilizando TSD, se han podido cuantificar algunos patrones de actividad observada. Por ejemplo, el grupo de investigación de Esther Thelen y Daniela Corbetta, ha desarrollado una teoría del aprendizaje motor en los infantes que lleva a un cuestionamiento radical de perspectivas clásicas. Siguiendo una metodología de la TSD, han 
identificado patrones característicos de la coordinación entre miembros durante ciertos periodos de desarrollo. Defienden que la coordinación y el control de un miembro, superior o inferior, no se limita a un procesador central o al desarrollo unilateral de ese miembro por sí solo. A través de diversos estudios $^{4}$ corroboran la idea sistémica de Bernstein respecto a que cualquier acto o habilidad motora es un sentido del cuerpo entero en cada acto: caminar o agarrar un juguete provienen de procesos complejos entre múltiples sistemas (Corbetta \& Thelen 2013). A raíz de sus hallazgos, plantean que la adquisición de la nueva habilidad motora ocurre posterior a la observación de patrones de progresión inestable. Es decir, que cuando en los registros aparecen patrones de transición, es el momento en el que se crea la plataforma de inestabilidad necesaria para el aprendizaje. En términos comportamentales esto se observa como cambios o fluctuaciones en la coordinación entre miembros motores, y muestra la tendencia hacia un nuevo patrón de comportamiento, como aprender a agarrar un juguete con una sola mano, sentarse, gatear o caminar (Corbetta \& Thelen 2013, Thelen \& Corbetta 2002).

En una línea similar, Kelso (2014), es otro de los teóricos que propone modelos experimentales y dinámicos para explicar el mecanismo de la novedad práctica. Él parte por considerar que los sistemas cognitivos motores de un agente humano son paisajes de atractores con distintos estadios de estabilidad, lo que corresponde a las disposiciones pre-existentes de los diferentes sistemas involucrados (nervioso, musculoesquelético) y de las repercusiones del medio. En su propuesta, la adquisición de aprendizaje es producto de un nuevo patrón motor que re-organiza todo el paisaje. En términos diagramáticos se observa como una transición (ruta de bifurcación inestable) que cambia las coordinaciones y estabilidades. Es decir, como en el caso de Thelen y Corbetta, y otros como Van Geert (2000, 2003) o Van Dijk et al. (2013), concuerdan que la generación de una novedad aprendida es la adquisición de estabilidad posterior a una transición inestable.

La definición operacional de novedad práctica propuesta, está asociada a este tipo de acercamientos teóricos y experimentales. Una vez que aceptamos que la novedad motora es un ensamble cooperativo de múltiples componentes, incluyendo aquellos que son parte del organismo humano y aquellos que constituyen el ambiente cambiante en el cual reside el organismo, se está haciendo un compromiso epistémico relacional que va delineando una respuesta a los problemas que nos interesan. Sin embargo, antes es necesario describir más profundamente los conceptos utilizados que juegan un papel central en mi propuesta.

\subsection{TSD en la novedad práctica y complejidad}

Un sistema dinámico es cualquier conjunto de fenómenos o componentes relacionados entre ellos, que cambian en el tiempo al ser afectados por otros sistemas, componentes, el ambiente o por ellos mismos (van Geert 2000, 2003). Son sistemas que evolucionan hacia alguna forma de estabilidad que puede ser medida con variables dinámicas y rangos temporales. Esto implica que tales sistemas son atraídos de manera espontánea hacia ciertos patrones como consecuencia de su dinámica propia, llamados atractores. Por ejemplo, algunos sistemas evolucionan hacia patrones que cambian de manera cíclica (como las oscilaciones van der Pol), o hacia un estado estable (atractor puntual), o hacia un estado caótico (atractor extraño). No obstante, cuando se está trabajando con sistemas dinámicos aplicados a agentes vivos y cognitivos, los atractores son menos regulares y las estabilidades más difíciles de encontrar. En cuanto al tema que nos compete, la emergencia de un patrón novedoso puede pensarse como un atractor inestable que motiva la generación de una nueva estabilidad.

La TSD afirma que, a pesar de que las relaciones iniciales entre variables podrían parecer deterministas y simples, existe una relación no-lineal ${ }^{5}$ y compleja (Ennis 1992). Modela los procesos de la cognición como coordinaciones e interacciones que cambian con el tiempo. Así la TSD, se vuelve

\footnotetext{
${ }^{4}$ Sus hallazgos preliminares indican que la transición de la búsqueda de dos manos a una mano ocurre en diferentes tiempos del desarrollo y varía entre infantes. Las variables que midieron de la dinámica de coordinación, es un análisis de movimiento basado en el perfil de velocidades de movimiento de ambas manos (incremento o disminución) (Corbetta \& Thelen 2013).

${ }^{5}$ No-linearidad implica que el éxito de una predicción disminuye conforme pasa el tiempo, porque la oportunidad para que ocurran pequeños cambios se va acumulando. El comportamiento futuro es cada vez más difícil de predecir por las interacciones continuas e inesperadas (Thelen \& Corbetta 2002).
} 
una buena herramienta para describir las variaciones sustanciales en la adquisición de nuevos aprendizajes prácticos de un agente humano en contextos particulares. En particular, desde este marco, se posibilita describir la emergencia de un nuevo patrón como resultado de interacciones no-lineales entre los componentes del sistema, entre los que se encuentran los constreñimientos biológicos del agente y su ambiente físico y social (Corbetta \& Thelen 2013, Van Dijk et al. 2013). Pero, ¿qué sucede en el sistema cuando emerge un nuevo atractor y se genera una nueva estabilidad? Bajo mi punto de vista, la auto-organización puede ser un buen candidato para explicar lo que acontece.

\subsection{Auto-organización en la novedad práctica}

La auto-organización es una condición necesaria para que emerja un nuevo patrón y para lograr una nueva estabilidad. Dicho de otra manera, la adquisición de un nuevo aprendizaje práctico depende de esta característica de los sistemas vivos.

La auto-organización es un fenómeno dinámico en el cual un alto número de unidades individuales espontáneamente generan una correlación irreducible que es sostenida de manera conjunta: i.e., un patrón colectivo de orden o de comportamiento que involucra todas aquellas unidades interactuantes y no puede ser explicado solo desde sus propiedades individuales. (...) Ocurre de manera espontánea, sin seguir una meta externa, plan o diseño, y cuando los procesos de asociación de esas partes no implican cambios de su identidad (Ruiz-Mirazo 2013).

Así, en los sistemas vivos, los múltiples procesos se auto-organizan a distintos niveles para producir patrones de comportamiento complejos a través de la cooperación mutua de las partes involucradas. Esto ocurre sin la necesidad de una estructura pre-existente o comando que ordene a todas las piezas funcionar de manera conjunta. No hay un procesador central, ni un conjunto de instrucción o diseño pre-existente, ni tampoco un elemento externo que sea privilegiado sobre los otros.

Al describir un sistema auto-organizado en términos de TSD, éste suele ser muy estable a lo largo de varios parámetros, pero puede exhibir cambios abruptos por consecuencia de cambios mínimos de uno o varios parámetros, lo que lo mueve organizarse nuevamente del estado inestable a otro estado con diversas formas de estabilidad (Kelso 2014). Entonces, la generación de nuevos patrones y su posterior adquisición es una consecuencia de nuevas auto-organizaciones que suceden a través de inestabilidades espontáneas (atractores), los cuales se acoplan periódicamente para ganar una nueva estabilidad que perdura en el tiempo. En cuanto a la generación de nuevos patrones de autoorganización en una habilidad práctica, se debe entender como una ocurrencia que involucra distintos sistemas interactuando como: la activación y fortalecimiento de vías nerviosas y neurales, el fortalecimiento de los músculos, el control de la postura, las evocaciones de comportamientos motores previos. Es decir, son muchos los sistemas que interactúan y que se tienen que re-organizar como respuesta a la inestabilidad que se generó y para ganar una nueva estabilidad. Este no es un proceso sencillo, de hecho existe un costo energético importante: la auto-organización consume energía.

\subsection{Emergencia}

La emergencia es otro de los conceptos que se discute en la literatura de sistemas dinámicos y que es útil para nuestra propuesta. Se refiere a la creación de nuevos procesos o estructuras más allá de los procesos existentes, que se originan espontáneamente y que, en general, no son reducibles a algún tipo de suma de sus partes. Dado que estos procesos de cambio están auto-organizados y evolucionan en el tiempo, pueden describirse desde un nivel previo (estructural) y un nivel posterior (organizacional) donde emergen los nuevos procesos.

En el caso de la novedad práctica en el agente humano, podríamos suponer que algunos de los sistemas involucrados son el sistema nervioso y el sistema musculoesquelético. Donde podemos describir que los componentes del nivel estructural son procesos psico-electroquímicos del sistema nervioso, caracterizados por distintos potenciales de acción y comportamientos entre las neuronas y moto-neuronas. Se trata de la unidad estructural que exhibe una cierta estabilidad temporal. Mientras que el nivel organizacional que va más allá de una regularidad temporal y espacial, se trata de las 
relaciones, interacciones y transformaciones entre los componentes estructurales y con el entorno, así como los patrones emergentes a los que dan lugar. Así, cuando se trata de un patrón novedoso que emerge en el nivel organizacional, éste no está determinado únicamente por un re-arreglo o una recombinación de los componentes estructurales, sino también por la auto-organización que es consecuencia de relaciones internas y con el exterior. Es decir, éste patrón es parte de un proceso relacional cuya existencia es la base conceptual de las discontinuidades que se observan en el agente cognitivo en interacción, y cada vez que emerge promueve el surgimiento de nuevas relaciones.

De acuerdo a esta descripción, la emergencia es considerada como una relación entre las descripciones de modelos de la mente/sistema nervioso y musculoesquelético, y no entre las propiedades de una realidad ontológica (objetiva). Por eso no se está apelando a una emergencia ontológica si no a una forma de emergencia epistémica la cual se describe entre las relaciones entre niveles que co-emergen y se co-especifican (Bich 2012).

No se está siendo anti-realista sino que más allá de buscar los niveles fundamentales de descripción, se está trabajando con relaciones entre descripciones donde la producción de novedad no depende de la medida de un observable escogido a priori en la descripción inicial, sino de las relaciones entre las clases de observables que convergen en diferentes modelos (Pessa 1998). Dos tipos de relaciones descriptivas permiten esta distinción entre diferentes modos de caracterizar la emergencia de la novedad práctica:

1. Emergencia como la impredecibilidad de un fenómeno o comportamiento con respecto a sus condiciones iniciales o elementos básicos y

2. Emergencia como la no-deductibilidad de un fenómeno o comportamiento con respecto al modelo que describe la dinámica inicial del sistema o sus constituyentes básicos (Bich 2012, González 2013, Pessa 1998).

La no predictibilidad de la dinámica interna es divergente en cuanto al comportamiento predicho y al efectivamente observado del sistema en un cierto tiempo. La no-deducibilidad ocurre porque la novedad emergente del nivel organizacional no puede ser deducida desde el modelo que describe la dinámica inicial o nivel estructural. Así, lo que emerge como novedad necesita una nueva descripción, un nuevo modelo que no puede reducirse al inicial. ${ }^{6}$

De este modo, la emergencia de la novedad puede implicar límites epistémicos, dado que ambas características se refieren a la incapacidad del agente cognitivo para construir explicaciones completas de fenómenos complejos (Bedau 2008). Pero, de acuerdo al marco que se asume, ambas caracterizaciones se refieren a los modelos y a sus relaciones descriptivas. El problema ya no es ontológico, sino respecto a las descripciones epistemológicas y la necesidad de nuevos modelos.

\subsection{Propuesta}

La novedad práctica es entonces emergente a partir de los cambios espontáneos en los patrones que se miden en la interacción entre varios sistemas, lo que promueve una auto-organización particular que tiene nuevos componentes y que no puede explicarse a partir del nivel previo de patrones periódicos. A mí consideración el patrón emergente inestable se encuentra entre el nivel estructural estable y el nuevo nivel organizacional que, gradualmente, encuentra su estabilidad (nueva estabilidad en nueva organización). Es importante enfatizar este aspecto, aunque la emergencia es espontánea los cambios de un estado a otro para lograr la estabilidad del nuevo nivel son paulatinos para que las alteraciones cualitativas que se adquieren perduren en el tiempo y se pueda hablar de aprendizaje adquirido.

Entonces, la inestabilidad que propongo, que podría denominarse "inestabilidad promotora de novedad" (IPN), tiene las siguientes condiciones:

- provenir de la interacción de varios sistemas trabajando al unísono en un tiempo dado,

- representar un cambio en la periodicidad o estabilidad del estadio previo,

\footnotetext{
${ }^{6}$ Este tipo de fenómenos incluye la simetría espontánea descrita por la teoría de los campos cuánticos en física (Bitbol 2007, Pessa 1998).
} 
- encontrarse entre el orden y el desorden.

Las primeras dos condiciones ya han sido explicadas, y la tercera se refiere al tipo de descripción que pueden tener estas inestabilidades. Yo defiendo que no son caóticas, y que por lo tanto no pueden describirse como atractores extraños, como proponen explícitamente Sawada y Caley (1985) o Ennis (1992) o cómo podría entenderse en la propuesta de Thelen y Corbetta (2002) o de Kelso (2014). Obviamente tampoco ordenadas puesto que el cambio es un contraste (de lo ordenado a lo otro) y lo que se mide es un cambio desde la regularidad. Así, yo considero que tienen un carácter complejo al encontrarse entre el orden y el desorden. La IPN tiene la característica de ser compleja ${ }^{7}$ y esto favorece tres implicaciones. Primero, que el nuevo elemento emergente y complejo no surge por generación espontánea, sino que la formación de estructuras complejas proviene de sus partes interactuantes. El nuevo componente del nivel organizacional, no es pura novedad porque requiere de muchos componentes de niveles previos, pero al ocurrir la auto-organización el sistema adquiere una nueva estabilidad que es distinta al estadio previo. ${ }^{8}$ Esto subraya que la auto-organización solo sucede entre sistemas que comparten energía y metabolitos, y que es a partir de la actividad entre ellos que emergen otros elementos que también son producto de la interacción entre energía y metabolitos. En el próximo apartado desarrollo más ampliamente esta idea.

Segundo, cuando el agente es confrontado con una nueva habilidad existe un cierto desequilibrio al tratar de entender el nuevo patrón motor e integrarlo a su repertorio de comportamientos. Por ello, cada vez que se adquiere nuevo conocimiento se desestabiliza el sistema y se requiere de una reorganización y re-estabilización adicional. Sin embargo, se debe subrayar que el proceso de aprendizaje muestra cierta selectividad, no se adquiere cualquier patrón motor, muchas veces los agentes pueden responder a las experiencias que consideren más significativas, pero otras veces no. ${ }^{9}$ Esta idea permite plantear que así como hay atractores que pueden surgir por perturbaciones del contexto, como el caso del desarrollo proximal ${ }^{10}$ a causa de un experto que enseña al aprendiz, también hay constreñimientos no intencionados que influyen y median el proceso dinámico.

Tercero, la complejidad implica que la inestabilidad que se mide en el sistema es un factor de la dinámica interna del agente. Es decir, que se está cuantificando un cambio interno. No obstante, también se debe tomar en cuenta que se está partiendo de una epistemología relacional, de una interacción constante con el entorno. Por ello, la dependencia del contexto de aprendizaje es una variable co-determinante. En la explicación, debe considerarse que la inestabilidad puede ser parte del acoplamiento interactivo con una perturbación externa, como el entrenamiento normado de una disciplina particular, o también con perturbaciones internas, como el recuerdo de una práctica motora individual o el reconocimiento de una parte del cuerpo por el dolor en ella. Su descripción no puede reducirse a simples procesos causales dirigidos por condiciones únicamente internas o únicamente externas.

En cuanto a este último punto, la importancia del acoplamiento del agente con el contexto también da cuenta del aspecto normativo de la práctica que se aprende. Debo mencionar que la adquisición de un nuevo patrón auto-organizado puede implicar un aprendizaje adaptativo y útil, pero también existen nuevos patrones que ganan su estabilidad siendo maladaptativos o dañinos. Por ello, yo creo que la normatividad de las inestabilidades debe buscarse en el contraste con los consensos

\footnotetext{
7 La complejidad proviene de la observación respecto a que algunos sistemas exhiben la formación de estructuras complejas a pesar de que sus partes interactuantes siguen reglas simples. En la actualidad, es aceptado que la complejidad puede explicarse por procesos de autoorganización en los cuales la relación entre las partes es dinámica, contextual e interdependiente (y muchas veces bi-direccional).

8 Este aspecto lo plantea Van Geert (2003, p. 641): “el desarrollo (motor) implica un aspecto de predestinación -algo que se despliega que ya está ahí- pero también un aspecto de algo que está surgiendo que es más que la simple cobertura de lo que está ahí desde el comienzo".

9 Ennis (1992) sugiere que la relevancia está asociada con la presencia de aprendizajes previos similares, así como la habilidad y motivación del agente. Sin estos componentes críticos, las estructuras del aprendiz permanecen estables y el aprendizaje no ocurre.

${ }^{10}$ El desarrollo proximal es una conceptualización que propone Lev Vygotsky (1980), la cual explica cómo es que los infantes adquieren nuevas habilidades y conocimiento por la ayuda que alguien más le brinda (un experto o una persona más competente), y esa experiencia de aprendizaje se vuelve parte de su nivel de desarrollo.
} 
intersubjetivos de las habilidades o prácticas en el contexto donde se están aprendiendo, como se mencionó en apartados anteriores.

Por último, solo resta mencionar, que tanto las inestabilidades como estabilidades de los sistemas, son términos descriptivos de modelos matemáticos, empíricos y conceptuales de la cognición humana. Se trata de metodologías con sistemas dinámicos que resultan muy interesantes y útiles sobre todo cuando se pretende tomar en cuenta este tipo de epistemologías y los cambios en el tiempo, pero no deja de ser un modelo.

\section{La variable ontogenética}

La novedad práctica, proviene del proceso dinámico de interacción entre un agente con un cuerpo específico que tiene una historia de acoplamiento particular. Este proceso se despliega como una transacción continua entre el contexto y el cuerpo que cambian durante la interacción, lo que provee de nuevas condiciones para los siguientes pasos en el proceso. Así, una de las virtudes del marco explicativo que propongo, deviene en que se está tomando en cuenta que el agente humano vivo se encuentra en cambio constante. La identidad de un agente está en desarrollo, se define por los procesos de auto-producción y auto-organización que más que resultar en una identidad inmutable, le ofrecen una identidad que varía con el tiempo.

En este aspecto, la conceptualización de identidad cuantitativa de Noonan (2009) y Razeto-Barry (2012) llama la atención. La "identidad numérica" o "identidad cuantitativa" se refiere al mantenimiento de una unidad discreta espacio-temporal sin perder su continuidad o incrementar o disminuir su número o cantidad, a pesar de que sus propiedades o cualidades sí pueden cambiar. Así, la única condición del agente humano para considerarse una unidad, es el hecho de mantener sus componentes en proximidad física, lo que permite la continua auto-producción química que lo mantiene vivo y asegura su continuidad espacio-temporal (Razeto-Barry 2012). Estas reacciones químicas se están modificando constantemente, porque los componentes involucrados y sus propiedades cualitativas cambian en el tiempo, sino fuera así, el ser humano tendría no se desarrollaría y probablemente no sobreviviría. No hay que olvidar que los sistemas vivos tienen ontogenia, una historia individual de acoplamiento, un crecimiento y también un desarrollo que varían a través del tiempo y los lugares de interacción.

Razeto-Barry (2012) propone que los sistemas auto-organizados no son sistemas que producen o mantienen sus propiedades constantes, porque de ser así los sistemas no cambiarían sus propiedades o procesos durante toda su vida. Más bien, los sistemas auto-organizados no mantienen una homeostasis organizacional (cualitativa) sino una homeostasis de su unidad individual (cuantitativa): donde los procesos y las propiedades cambian y se renuevan. Es por esto, que la identidad humana puede pensarse de un modo diacrónico, al cambiar a través del tiempo (Noonan 2009).

Cuando se plantea que el desarrollo puede describirse como un progreso continuo de estadios secuenciales que están inevitablemente dirigidos hacia la adquisición de una función jerárquicamente mayor, no se está reparando en los detalles de la co-dependencia entre un organismo que cambia y un entorno variable. En este artículo, el desarrollo de las especies en general se piensa como imbuido en los patrones de comportamiento que se auto-organizan y evolucionan en cada organismo durante toda su vida. Por ello, variables como los patrones de alimentación, de reproducción, de reparación de tejidos, o la correlación entre la edad y las capacidades plásticas y adaptativas del agente, se consideran importantes. Específicamente en el desarrollo humano, las capacidades fisiológicas, conductuales o comunicativas se consolidan con el pasar de los años y constriñen los tipos de procesos de aprendizaje y los tiempos en que puedan llevarse a cabo. Por ejemplo, la capacidad de adquirir prácticas motoras ocupa mucho menos tiempo en un infante pre-lingüístico que en un adulto que por alguna razón no la ha adquirido o la ha perdido.

La novedad práctica está constreñida por la historia de acoplamiento y por la etapa de desarrollo en la que se encuentre el agente que aprende. 


\section{Apuntes finales}

En las últimas décadas, las teorías del desarrollo y adquisición de la novedad práctica han cambiado de una perspectiva top-down que considera la maduración del cerebro actualizada año por año, a una perspectiva más contemporánea donde las causas dejan de considerarse estructuradas únicamente a partir de mecanismos y descomponibles en términos unidireccionales. Este giro, permite que surjan propuestas que parten de epistemologías menos tradicionales, donde la continua interacción con el contexto y las etapas ontogenéticas del agente son descripciones necesarias. Este es el caso del planteamiento de este texto.

La introducción de un pensamiento emergentista y auto-organizado, me permitió plantear una descripción de los procesos que dan lugar a elementos nuevos en el nivel organizacional y de la importancia del contexto no como causa única sino como co-determinante. Se ha defendido que el aprendizaje de nuevas habilidades motoras, lo que yo he denominado novedad práctica, puede entenderse desde el marco explicativo propuesto y posibilita abrir nuevas vías de investigación. Asimismo, promover la creación de modelos más particulares para entender más propiedades de estos patrones emergentes o elementos nuevos. Una de las ventajas de esta forma de naturalizar la novedad práctica, es que otorga la posibilidad de modelar experimentalmente el proceso de adquirir la novedad dentro de contextos culturales diversos.

La epistemología propuesta me parece más adecuada y con menos requerimientos ontológicos que otras perspectivas que se vuelven difíciles de sostener cuando se contrasta con una base empírica naturalista. Igualmente, la normatividad de dos aspectos, en la cual las normas no están dadas por criterios de buen razonamiento que provienen de las reglas de la lógica deductiva o el cálculo de probabilidades; sino que se encuentra en la interfaz entre los consensos intersubjetivos de lo que es una práctica adecuada e innovadora en un contexto dado y las posibilidades de acción del cuerpo biológico (o artificial)

Por último, considero que esta manera de entender la adquisición de novedad práctica es prometedora al tomar en cuenta que los sistemas cambian en el tiempo por la ontogenia que los caracteriza. La TSD es una buena herramienta para explicar el intrincado proceso de la novedad que actúa a diferentes niveles de cambio y complejidad. Aunque es factible pensar que distintos enfoques teóricos podrían dar cuenta de mejor manera de los diferentes tipos de novedad, quizás los enfoques tradicionales pueden ser más útiles para el tipo de novedad relacionado con el conocimiento proposicional, mientras que propuesta dinámicas para la novedad práctica. Todavía quedan muchas tareas por hacer, por mi parte mi intención era mostrar algunas vías de solución a la paradoja del aprendizaje al introducir un marco epistémico, proponer un modelo explicativo e identificar algunas de las variables involucradas.

Bibliografía

Bedau, M. (2008), “Is Weak Emergence Just in the Mind?”, Minds Eु Machines 18: 443-459.

Bich, L. (2012), “Complex Emergence and the Living Organization: An Epistemological Framework for Biology”, Synthese 185: 215-232.

Bitbol, M. (2007), “Ontology, Matter and Emergence”, Phenomenology and the Cognitive Science 6: 293-307.

Boom, J. (2012), "Collective Development and the Learning Paradox", en Seel, N.M. (ed.), Encyclopedia of the Sciences of Learning, Boston: Springer, pp. 643-646.

Brewer, B. (2006), "Perception and Content”, European Journal of Philosophy 14(2): 165-181.

Burnet, J. (1920), Early Greek Philosophy, 3rd edition, London: Adam \& Charles Black. 
Corbetta, D. y E. Thelen (2013), "Interlimb Coordination in Infant's Reaching”, en Swinnen, S.P., Massion, J., Heuer, H. y P. Casaer (eds.), Interlimb Coordination: Neural, Dynamical, and Cognitive Constraints, San Diego: Academic Press, pp. 413-438.

Dreyfus, H.L. (2002), “Intelligence without Representation”, Phenomenology and the Cognitive Sciences 1: 367-383.

Ennis, C. (1992), "Reconceptualizing Learning as a Dynamical System”, Journal of Curriculum and Supervision 7(2):115130.

Fodor, J. (1980), "Fixation of Belief and Concept Acquisition”, en Piattelli-Palmarini, M. (ed.), Language and Learning: The Debate between Jean Piaget and Noam Chomsky, Cambridge: Harvard University Press.

Fodor, J. (1981), “The Present Status of the Innateness Controversy”, en Fodor, J. (ed.), Representations, Brighton: Harvester Press, pp. 257-316.

Gesell, A. (1946), The Ontogenesis of Infant Behavior, New York: Wiley.

Gibson, J.J. (1977), “The Theory of Affordances”, en Shaw, R. y J. Bransford (eds.), Perceiving, Acting, and Knowing: Toward an Ecological Psychology, Hillsdale, NJ: Lawrence Erlbaum, pp. 67-82.

González, X. (2013), “La auto-producción de la subjetividad: autopoiesis y cognición de alto nivel”, en Razeto-Barry, P. y R. Ramos (eds.), Autopoiesis. Un concepto vivo, Santiago de Chile: Universitas Nueva Civilización, pp. 95-118.

Hofsten, C. y S. Fazel-Zandy (1984), "Development of Visually Guided Hand Orientation in Reaching”, Journal of Experimental Child Psychology 38: 208-219.

Hurley, S. (2008), "The Shared Circuits Model: How Control, Mirroring and Simulation can Enable Imitation and Mindreading", Behavioral and Brain Sciences 31: 1-58.

Kelso, J.A. (2014), “The Dynamic Brain in Action: Coordinative Structures, Criticality, and Coordination Dynamics”, en Plenz, D. y E. Niebur (eds.), Criticality in Neural Systems, Mannheim: John Wiley \& Sons, pp. 67-104.

Mazur, J.E. y R. Hastie (1978), "Learning as Accumulation: A Reexamination of the Learning Curve”, Psychological Bulletin 85(6): 1256-1274.

McDowell, J. (1994), Mind and World, Cambridge: Harvard University Press.

Merleau-Ponty, M. (1975), Fenomenología de la percepción, Barcelona: Península.

Noonan, H. (2009), "Identity", en Zalta, E.N. (ed.), The Stanford Encyclopedia of Philosophy, URL = http://plato.stanford.edu/entries/identity/.

Pessa, E. (1998), “Emergence, Self-Organization, and Quantum Theory”, en Minati, G. (ed.), First Italian Conference on Systemics, Milano: Apogeo, pp. 59-80.

Piaget, J. (1959), The Language and Thought of the Child, London: Psychology Press.

Platón (1871), "Menón o de la virtud”, en Azcárate, P. de (ed.), Platón, Obras completas, tomo 4, Madrid: Medina y Navarro, pp. 275-345.

Razeto-Barry, P. (2012) “Autopoiesis 40 Years Later. A Review and a Reformulation”, Origins of Life and Evolution of Biospheres 42(6): 543-567.

Rochat, P. (1992), "Self-Sitting and Reaching in 5 to 8 Month Old Infants: The Impact of Posture and its Development on Early Eye-Hand Coordination”, Journal of Motor Behavior 24: 210-220.

Ruiz-Mirazo, K. (2013), “Self-Organization”, en Dubitzky, W., Wolkenhauer, O., Cho, K.H. y H. Yokota (eds.), Encyclopedia of Systems Biology, New York: Springer, pp. 1915-1919.

Ryle, G. (1946), "Knowing How and Knowing That”, Proceedings of the Aristotelian Society 46: 1-16.

Sawada, D. y M.T. Caley (1985), “Dissipative Structures: New Metaphors for Becoming in Education”, Educational Researcher 14(3): 13-19.

Siegler, R.S. (1996), Emerging Minds: The Process of Change in Children's Thinking, New York: Oxford University Press.

Thelen, E. y D. Corbetta (2002), "Microdevelopment and Dynamic Systems: Applications to Infant Motor Development”, en Granott, N. y J. Parziale (eds.), Microdevelopment. Transition Processes in Development and Learning, New York: Cambridge University Press, pp. 59-79.

Toribio, J. (2008), “How Do We Know How?”, Philosophical Explorations 11(1): 39-52. 
Van Dijk, M., Van Geert, P., Korecky-Kröll, K., Maillochon, I., Laaha, S., Dressler, W.U. y D. Bassano (2013), "Adaptation between Child-Directed Speech and Early Child Language: A Developmental Perspective", Language Learning 63: 214-270.

Van Geert, P. (2000), "The Dynamics of General Developmental Mechanisms from Piaget and Vygotsky to Dynamic Systems Models", Current Directions in Psychological Science 9(2): 64-68.

Van Geert, P. (2003), "Dynamic Systems Approaches and Modeling of Developmental Processes", en Valsiner, J. y K.J. Connolly (eds.), Handbook of Developmental Psychology, New York: Sager, pp. 640-672.

Varela, F.J., Thompson, E. y E. Rosch (1991), The Embodied Mind: Cognitive Sciences and Human Experience, Massachussets: MIT Press.

Viale, R. (2013), "Art and Science: Some Neurocognitive Remarks on Creativity Creativity Art", en Viale, R. (ed.), Methodological Cognitivism, Heidelberg: Springer, pp. 197-216.

Vygotsky, L.S. (1980), Mind in Society: The Development of Higher Psychological Processes, Harvard: Harvard University Press.

Witt, U. (2005), On Novelty and Heterogeneity, Heidelberg: Springer.

Wolpert, D.M., Ghahramani, Z. y J.R. Flanagan (2001), "Perspectives and Problems in Motor Learning", Trends in Cognitive Sciences 5: 487-494. 